\title{
Perfiles de resistencia a fluoroquinolonas en aislamientos clínicos de cocos Gram positivos provenientes de hospitales colombianos, 1994-2004
}

\author{
Marylin Hidalgo', Jinnethe Reyes², Ana María Cárdenas', Lorena Díaz², Sandra Rincón², \\ Natasha Vanegas ${ }^{3}$, Paula Lucía Díaz', Elizabeth Castañeda' ${ }^{1}$, César A. Arias²,4 \\ ${ }^{1}$ Grupo de Microbiología, Instituto Nacional de Salud, Bogotá, D. C., Colombia \\ 2 Unidad de Genética y Resistencia Antimicrobiana, Universidad El Bosque, Bogotá, D. C., Colombia \\ ${ }^{3}$ Instituto de Genética Molecular Bacteriana, Universidad El Bosque, Bogotá, D. C., Colombia \\ ${ }^{4}$ Division of Infectious Diseases, University of Texas Medical School, Houston, Texas, USA \\ Nota: Marylin Hidalgo y Jinnethe Reyes contribuyeron de igual forma en este estudio
}

Introducción. Las fluoroquinolonas son antibióticos de amplio espectro comúnmente utilizados en el manejo de infecciones.

Objetivo. Determinar los patrones de resistencia a fluoroquinolonas en aislamientos colombianos de Streptococcus pneumoniae, Staphylococcus aureus, estafilococos coagulasa negativa y enterococos de centros hospitalarios colombianos, recolectados entre 1994 y 2004. Materiales y métodos. Se realizó la determinación de concentraciones inhibitorias mínimas frente a ciprofloxacino, moxifloxacino y gatifloxacino a 270 aislamientos clínicos de $S$. pneumoniae, 348 de $S$. aureus, 176 de estafilococos coagulasa negativa y 123 de enterococos. Para los aislamientos de $S$. aureus resistentes a la meticilina se determinó la concentración inhibitoria mínima frente a levofloxacino y para aislamientos de $S$. pneumoniae se realizó la prueba de difusión en agar con discos de ofloxacino y levofloxacino.

Resultados. Doscientos sesenta y nueve $(99,6 \%)$ aislamientos de $S$. pneumoniae fueron susceptibles a gatifloxacino y moxifloxacino; para levofloxacino y ofloxacino la resistencia fue del $1,5 \%$ y $8,9 \%$, respectivamente. El $15,9 \%$ de $S$. pneumoniae tuvo una concentración inhibitoria mínima $\geq 4 \mu \mathrm{g} / \mathrm{ml}$ frente a ciprofloxacino. Las prevalencias de resistencia para ciprofloxacino, gatifloxacino y moxifloxacino en los 348 aislamientos de $S$. aureus fueron $55,4 \%, 54,9 \%$ y $52,6 \%$, y en $S$. aureus resistentes a meticilina, fueron $92,3 \%, 91,3 \%$ y $87,5 \%$ y $91,8 \%$ para levofloxacino. En estafilococos coagulasa negativa y enterococos susceptibles a vancomicina se observaron tasas de resistencia entre $25,6 \%$ y $31,8 \%$. Todos los aislamientos de enterococos resistente a vancomicina fueron resistentes a los compuestos evaluados.

Conclusión. Los aislamientos colombianos de $S$. pneumoniae mantienen susceptibilidad a las fluoroquinolonas de última generación. La resistencia a fluoroquinolonas es alta en $S$. aureus, especialmente en aislamientos resistentes a la meticilina (cercana al 100\%).

Palabras clave: fluoroquinolonas, Streptococcus pneumoniae, Staphylococcus aureus, Enterococcus, resistencia microbiana a los medicamentos, Colombia.

\section{Resistance profiles to fluoroquinolones in clinical isolates of Gram positive cocci}

Introduction. Fluoroquinolones are broad spectrum antibiotics commonly used in the treatment of infections.

Objective. Resistance profiles of coccus bacteria to fluoroquinolones were evaluated in isolates of Streptococcus pneumoniae, Staphylococcus aureus, coagulase negative staphylococci and Enterococcus spp. The samples were recovered from Colombian hospitals between 1994 and 2004.

Materials and methods. The minimal inhibitory concentration of ciprofloxacin, moxifloxacin and gatifloxacin was determined in 270 clinical isolates of $S$. pneumoniae, 348 of $S$. aureus, 176 of coagulase negative staphylococci and 123 of coagulase-negative enterococci. The minimal inhibitory concentration of levofloxacin was also determined in all isolates of methicillin- 
resistant $S$. aureus. An agar diffusion susceptibility test with disks of levofloxacin and ofloxacin was also applied to all isolates of $S$. pneumoniae.

Results. A total of 269 (99.6\%) isolates of S. pneumoniae were susceptible to moxifloxacin and gatifloxacin. For levofloxacin and ofloxacin, resistance in S. pneumoniae was found in $1.5 \%$ and $8.9 \%$ of isolates, respectively. The ciprofloxacin minimal inhibitory concentration was $\geq 4$ $\mu \mathrm{g} / \mathrm{ml}$ in $15.9 \%$ of pneumococcal isolates. The rates of resistance to ciprofloxacin, gatifloxacin and moxifloxacin in the $348 \mathrm{~S}$. aureus isolates were $55.4 \%, 54.9 \%$ and $52.6 \%$, respectively; increasing to $92.3 \%, 91.3 \%$ and $87.5 \%$, respectively in methicillin resistant isolates. Resistance to levofloxacin was found in $91.8 \%$ of MRSA isolates. The rates of resistance to ciprofloxacin, gatifloxacin and moxifloxacin in coagulase negative staphylococci and vancomycin-susceptible enterococci were between $25.6 \%$ and $31.8 \%$. All vancomycin-resistant enterococci were resistant to all fluroquinolones tested.

Conclusion. The newer fluoroquinolones have maintained effective activity against clinical isolates of $S$. pneumoniae. The rates of fluoroquinolone resistance in $S$. aureus were very high, particularly in methicillin resistant isolates (approaching 100\%).

Key words: fluoroquinolones, Streptococcus pneumoniae, Staphylococcus aureus, Enterococcus; drug resistance, microbial; Colombia.

Las fluoroquinolonas son antibióticos fluorinados sintéticos introducidos en la práctica clínica a mediados de los 80. Estos compuestos son derivados del ácido nalidíxico, introducido a la práctica clínica en 1962 (1). El espectro de actividad inicial contra Gram positivos era bastante pobre, pero las modificaciones de la estructura de la molécula han ampliado su actividad contra estos microorganismos. La nueva generación de fluoroquinolonas, que incluye el esparfloxacino, el grepafloxacino, el gatifloxacino y el moxifloxacino, tiene potente actividad contra cocos Gram positivos, inclusive S. pneumoniae (2-4).

Las fluoroquinolonas interfieren en la síntesis de ADN inhibiendo la acción de las enzimas girasa y topoisomerasa IV de ADN (3-6). Los mecanismos de resistencia a las fluoroquinolonas en cocos Gram positivos han sido extensamente caracterizados e incluyen: i) disminución en la acumulación del antibiótico dentro de la bacteria debido a la reducida permeabilidad de la membrana; ii) aumento de la expresión de las bombas de exclusión (como NorA), y iii) mutaciones en los

\footnotetext{
Correspondencia:

César A. Arias, Division of Infectious Diseases, University of Texas Medical School, 6431 Fannin St., MSB 2112, Houston, USA.

Teléfono: (713) 500 6765; fax: (713) 5005495

caa22@cantab.net
}

Recibido: 31/07/07; aceptado: 20/11/07 genes que codifican para las subunidades de las enzimas girasa ( $g y r A$ y gyrB) y topoisomerasa IV de ADN (parC y parE, también denominadas grlA y grlB en Staphylococcus aureus), que tienden a agruparse en dominios muy conservados denominadas regiones QRDR (quinolone resistance determining region) (3-6).

Recientemente, se reportó en Enterococcus faecalis la presencia del gen qnr, cuyo producto protege la girasa de ADN de la inhibición por fluoroquinolonas, mecanismo que se había descrito únicamente en bacterias Gram negativas en el pasado (7-9).

En Colombia, un estudio multicéntrico realizado en cinco ciudades indicó que el porcentaje de resistencia a ciprofloxacino en estafilococos y enterococos era de $25 \%$ y $83 \%$, respectivamente (10). La resistencia a ciprofloxacino reportada por el Grupo para el Control de la Resistencia en Bogotá (GREBO) para los años 2001 a 2003, en unidades de cuidados intensivos de hospitales de tercer nivel de atención en Bogotá, estuvo entre $60 \%$ y $63 \%$ para $S$. aureus, entre $47 \%$ y $58 \%$ para estafilococos coagulasa negativa y entre $25 \%$ y $44 \%$ para enterococos (11).

Sin embargo, hasta el momento no se ha realizado un estudio sistemático que evalúe la prevalencia de resistencia a las fluoroquinolonas de última generación en cocos Gram positivos en Colombia y los datos en neumococo (uno de los principales 
blancos en la terapia con fluoroquinolonas) son limitados.

El objetivo de este trabajo fue determinar los perfiles de resistencia a las fluoroquinolonas, incluyendo compuestos de nueva generación, en aislamientos colombianos de $S$. pneumoniae, $S$. aureus, estafilococcos coagulasa negativa y Enterococcus spp., recuperados a través de programas multicéntricos de vigilancia de resistencia antibiótica a estos microorganismos entre 1994 y 2004.

\section{Materiales y métodos}

\section{Aislamientos bacterianos}

Streptococcus pneumoniae. Se estudiaron 270 aislamientos clínicos provenientes de sangre (84\%) y líquido pleural (16\%), recuperados de pacientes adultos con diagnóstico de neumonía, que fueron enviados al Grupo de Microbiología del Instituto Nacional de Salud como parte del programa de vigilancia por el laboratorio de la infección respiratoria aguda (IRA) entre 1994 y 2004.

La procedencia de los aislamientos fue la siguiente: 96 de 22 hospitales de Bogotá, 91 de 10 hospitales de Antioquia, 36 de cuatro hospitales del Valle del Cauca, 22 de cuatro hospitales de Santander, 21 de tres hospitales de Risaralda, y 1 aislamiento de un hospital en Caldas, Meta, Norte de Santander y Amazonas, respectivamente (12).

Los aislamientos se conservaron en leche descremada al $20 \%$ a $-70^{\circ} \mathrm{C}$, fueron recuperados en agar sangre de cordero al $5 \%$ y confirmados con la susceptibilidad a optoquina y la solubilidad en bilis (13). Todos los aislamientos se serotipificaron por la técnica de Quellung y se les había determinado previamente la susceptibilidad antimicrobiana a penicilina, trimetoprimsulfametoxazol, ceftriaxona, cloranfenicol, tetraciclina y vancomicina $(12,14)$.

Staphylococcus aureus. Se evaluaron 348 aislamientos invasivos de $S$. aureus. De éstos, 208 eran resistentes a meticilina y 140 sensibles a meticilina. Ciento cincuenta y cinco aislamientos de $S$. aureus resistentes a la meticilina y todos los $S$. aureus sensibles a la meticilina se recolectaron entre marzo del 2001 y marzo de
2002, provenientes de 15 hospitales de tercer nivel en Colombia: ocho en Bogotá, tres en Cali, uno en Medellín, uno en Cartagena y dos en Bucaramanga. Los $53 \mathrm{~S}$. aureus resistentes a meticilina restantes se recuperaron a partir de cinco hospitales de Bogotá en el 2003. La confirmación de especie de estos aislamientos y la detección del gen de resistencia a meticilina $(m e c A)$ se realizó por ensayos de PCR múltiple, como se ha descrito anteriormente (15).

Estafilococos coagulasa negativa y Enterococcus spp. Los aislamientos de estafilococos coagulasa negativa y Enterococcus spp. se recolectaron entre marzo de 2001 y marzo de 2002, provenientes de 15 hospitales de tercer nivel en el país: ocho en Bogotá, tres en Cali, uno en Medellín, uno en Cartagena y dos en Bucaramanga. Se analizaron 176 aislamientos clínicos invasivos de estafilococos coagulasa negativa, de los cuales, $121(69 \%)$ correspondieron a S. epidermidis y fueron confirmados por ensayos de PCR múltiple y la detección del gen de resistencia a meticilina ( $m e c A)(74,4 \%$ fueron resistentes a meticilina) (15). El restante $31 \%$ correspondió a estafilococos coagulasa negativa no epidermidis. Los estafilococos coagulasa negativa no epidermidis se identificaron por sistemas automatizados (Vitek I y Microscan) como $S$. haemolyticus (10\%), $S$. hominis (5\%) y, en menor proporción, S. warneri, S. simulans, S. capitis, S. saprophyticus, $S$. auricularis y $S$. sciuri.

Se estudiaron 123 aislamientos de enterococos, de los cuales, 101 se identificaron como $E$. faecalis, 17 como $E$. faecium, dos se identificaron como E. avium, uno como E. durans y dos como E. hirae, utilizando ensayos de PCR múltiple para identificación de especies como se describió previamente $(16,17)$.

Para los aislamientos de $S$. aureus y estafilococos coagulasa negativa se habían determinado previamente los perfiles de susceptibilidad frente a oxacilina, eritromicina, clindamicina, tetracilina, cloranfenicol, gentamicina, vancomicina, teicoplanina, linezolid, rifampicina y trimetoprimsulfametoxazol, y para los aislamientos de enterococo, frente a ampicilina, cloranfenicol, vancomicina, teicoplanina, linezolid y determinación 
de altos niveles de resistencia a gentamicina y estreptomicina $(10,18,19)$.

\section{Pruebas de susceptibilidad antimicrobiana}

La determinación de la susceptibilidad antimicrobiana por microdilución en caldo frente a ciprofloxacino (ICN; USA), moxifloxacino (Bayer, Alemania), gatifloxacino (Bristol Myers Squibb, USA) y levofloxacina (Johnson and Johnson Pharmaceutical Research and Development) para $S$. aureus resistentes a meticilina, se realizó bajo las recomendaciones del Clinical Laboratory Standards Institute (CLSI) (20).

La determinación de la susceptibilidad frente a ofloxacina y levofloxacina en S. pneumoniae se realizó con la técnica de difusión en agar KirbyBauer empleando discos de ofloxacina y levofloxacina de $5 \mu \mathrm{g}$ (BBL, Becton Dickinson and Company, Franklin Lakes, NJ, USA). La interpretación de los halos de inhibición se realizó siguiendo las recomendaciones del CLSI (21). Se emplearon como control las cepas de S. pneumoniae ATCC 49619, S. aureus 29213 y E. faecalis 29212. Es importante resaltar que el CLSI no tiene puntos de corte definidos para el ciprofloxacino en $S$. pneumoniae. Sin embargo, en varios estudios se interpreta como resistentes a ciprofloxacino aislamientos con una concentración inhibitoria mínima $(\mathrm{CIM}) \geq 4 \mu \mathrm{g} / \mathrm{ml}(22-26)$.

\section{Resultados}

\section{Streptococcus pneumoniae}

De los 270 aislamientos clínicos de S. pneumoniae sólo uno presentó resistencia al gatifloxacino $(\mathrm{CIM}=8 \mu \mathrm{g} / \mathrm{ml})$ y al moxifloxacino $(\mathrm{CIM}=4 \mu \mathrm{g} / \mathrm{ml})$. El resto de los aislamientos $(99,6 \%)$ mostraron susceptibilidad a estos antibióticos. La $\mathrm{CIM}_{90}$ para el moxifloxacino fue $0,25 \mu \mathrm{g} / \mathrm{ml}$ y para el gatifloxacino, $0,5 \mu \mathrm{g} / \mathrm{ml}$ (cuadro 1). Para la levofloxacina y la ofloxacina la resistencia fue de $1,5 \%$ y $8,9 \%$, respectivamente. Se observó una mejor actividad in vitro de moxifloxacino comparada con la de gatifloxacino (cuadro 1), como se ha reportado previamente (22). Es importante resaltar que el $15,9 \%$ de los aislamientos tuvo una CIM para ciprofloxacino $\geq 4$ $\mu \mathrm{g} / \mathrm{ml}$. No se observó ninguna diferencia en la actividad de las fluoroquinolonas evaluadas, frente a los aislamientos con susceptibilidad disminuida a la penicilina y los aislamientos sensibles.

\section{Staphylococcus aureus y estafilococos coagulasa negativa}

Las tasas de resistencia para ciprofloxacino, gatifloxacino y moxifloxacino en los 348 aislamientos de $S$. aureus fueron $55,4 \%, 54,9 \%$ y $52,6 \%$, respectivamente. La alta resistencia a las fluoroquinolonas fue evidente en los aislamientos de $S$. aureus resistentes a la

Cuadro 1. Distribución de concentraciones inhibitorias mínimas para fluoroquinolonas en S. pneumoniae.

\begin{tabular}{|c|c|c|c|c|c|c|c|c|c|c|c|c|}
\hline \multirow[t]{2}{*}{$\begin{array}{l}\text { Microorganismos } \\
\text { (n) }\end{array}$} & \multirow[t]{2}{*}{ Antibiótico* } & \multicolumn{5}{|c|}{ Número de aislamientos } & \multirow{2}{*}{$\begin{array}{c}\text { con } \\
1\end{array}$} & \multicolumn{2}{|c|}{ su respectiv } & \multicolumn{3}{|c|}{$\operatorname{CIM}(\mu \mathrm{g} / \mathrm{ml})$} \\
\hline & & $\leq 0,03$ & 0,06 & 0,12 & 0,25 & 0,5 & & 2 & 4 & 8 & $\geq 16$ & $\% \mathbf{R}$ \\
\hline S. pneumoniae (270) & CIP & - & - & - & - & 20 & 110 & 97 & 42 & - & 1 & NA \\
\hline GAT & 1 & 1 & 21 & 137 & 102 & 7 & - & $=$ & 1 & - & 0,4 & \\
\hline MOX & - & 14 & 193 & 59 & 3 & - & - & $\underline{1}$ & - & - & 0,4 & \\
\hline S. pneumoniae & $\mathrm{CIP}$ & - & - & - & - & - & 32 & 25 & 12 & - & - & NA \\
\hline resistente a & GAT & - & - & 7 & 41 & 20 & 1 & - & $=$ & - & - & 0 \\
\hline penicilina (69) & MOX & - & 5 & 52 & 12 & - & - & - & $=$ & - & - & 0 \\
\hline S. pneumoniae & CIP & - & - & - & - & 20 & 78 & 72 & 30 & - & 1 & NA \\
\hline susceptible a & GAT & 1 & 1 & 14 & 96 & 82 & 6 & - & $=$ & 1 & - & 0,5 \\
\hline penicilina (201) & MOX & - & 9 & 141 & 47 & 3 & - & - & $\overline{1}$ & - & - & 0,5 \\
\hline
\end{tabular}

Subrayado: puntos de corte $(\mu \mathrm{g} / \mathrm{ml})$; negrilla: $\mathrm{CIM}_{90}$ ${ }^{*} \mathrm{CIP}$ : ciprofloxacino; GAT: gatifloxacino; MOX: moxifloxacino NA: no aplica 
meticilina, en los que las tasas de resistencia fueron del $92,3 \%, 91,8 \%, 91,3 \%$ y $87,5 \%$ para ciprofloxacino, levofloxacina, gatifloxacino y moxifloxacino, respectivamente (cuadro 2).

Estas tasas de resistencia en aislamientos de $S$. aureus resistentes a meticilina fueron considerablemente mayores que las de $S$. aureus susceptibles a meticilina (inferiores al $1 \%$ con una $\mathrm{CIM}_{90}$ de $1 \mu \mathrm{g} / \mathrm{ml}$ para ciprofloxacino, $0,5 \mu \mathrm{g} / \mathrm{ml}$ para gatifloxacino y $0,25 \mu \mathrm{g} / \mathrm{ml}$ para moxifloxacino). Además, es importante resaltar que estos aislamientos de $S$. aureus resistentes a la meticilina son multirresistentes, con altas tasas de resistencia (mayor del $80 \%$ ) frente a eritromicina, clindamicina y gentamicina, comparadas con la resistencia a estos antimicrobianos en $S$. aureus susceptibles a meticilina.

En estafilococos coagulasa negativa se observó una resistencia de $31,8 \%, 30,1 \%$ y $25,6 \%$ a ciprofloxacino, gatifloxacino y moxifloxacino, respectivamente. Estas tasas de resistencia aumentaron en los aislamientos de $S$. epidermidis resistentes a la meticilina), en los cuales la resistencia frente a ciprofloxacino, gatifloxacino y moxifloxacino fue de $43,3 \%, 40 \%$ y $34,4 \%$, respectivamente (cuadro 2). De las tres fluoroquinolonas evaluadas, moxifloxacino tuvo una mejor actividad en comparación con ciprofloxacino y gatifloxacino.

\section{Enterococcus spp.}

En los 123 aislamientos de enterococos, la resistencia a fluoroquinolonas fue del $30 \%$ para ciprofloxacino, $28 \%$ para gatifloxacino y $26 \%$ para moxifloxacino. Los aislamientos de E. faecium mostraron unas tasas de resistencia más altas comparadas con las de los aislamientos de $E$. faecalis (64\% Vs. $25 \%$ para ciprofloxacino, $52,9 \%$ Vs. $24 \%$ para gatifloxacino y $52,9 \%$ Vs. $22 \%$ para

Cuadro 2. Distribución de concentraciones inhibitorias mínimas para fluoroquinolonas en estafilococos.

\begin{tabular}{|c|c|c|c|c|c|c|c|c|c|c|c|c|c|c|}
\hline \multirow[t]{2}{*}{$\begin{array}{l}\text { Microorganismos } \$ \\
\text { (n) }\end{array}$} & \multirow[t]{2}{*}{ Antibiótico* } & \multicolumn{13}{|c|}{ Número de aislamientos con su respectiva $\mathrm{CIM}(\mu \mathrm{g} / \mathrm{ml})$} \\
\hline & & $\leq 0,03$ & 0,06 & 0,12 & 0,25 & 0,5 & 1 & 2 & 4 & 8 & 16 & 32 & $\geq 64$ & $\% \mathbf{R}$ \\
\hline S. aureus (348) & $\begin{array}{l}\text { CIP } \\
\text { GAT } \\
\text { MOX }\end{array}$ & $\begin{array}{c}2 \\
1 \\
14\end{array}$ & $\begin{array}{c}2 \\
10 \\
69\end{array}$ & $\begin{array}{l}13 \\
93 \\
43\end{array}$ & $\begin{array}{l}52 \\
25 \\
17\end{array}$ & $\begin{array}{l}68 \\
26 \\
12\end{array}$ & $\begin{array}{c}17 \\
2 \\
10\end{array}$ & $\begin{array}{c}1 \\
\underline{24} \\
\underline{118} \\
\end{array}$ & $\begin{array}{c}\frac{1}{143} \\
58\end{array}$ & $\begin{array}{c}10 \\
23 \\
7\end{array}$ & $\begin{array}{c}96 \\
1 \\
-\end{array}$ & $\begin{array}{c}60 \\
- \\
-\end{array}$ & $\begin{array}{c}26 \\
- \\
-\end{array}$ & $\begin{array}{l}55,4 \\
54,9 \\
52,6\end{array}$ \\
\hline SARM (208) & $\begin{array}{l}\text { CIP } \\
\text { GAT } \\
\text { MOX } \\
\text { LVX }\end{array}$ & $\begin{array}{l}- \\
- \\
- \\
-\end{array}$ & $\begin{array}{l}- \\
- \\
7 \\
-\end{array}$ & $\begin{array}{c}1 \\
11 \\
4 \\
3\end{array}$ & $\begin{array}{l}4 \\
2 \\
2 \\
7\end{array}$ & $\begin{array}{l}9 \\
3 \\
3 \\
5\end{array}$ & $\begin{array}{c}2 \\
2 \\
10 \\
2\end{array}$ & $\begin{array}{c}- \\
\frac{24}{117} \\
-\end{array}$ & $\begin{array}{c}\frac{1}{142} \\
58 \\
\underline{8}\end{array}$ & $\begin{array}{c}10 \\
\mathbf{2 3} \\
7 \\
127\end{array}$ & $\begin{array}{c}96 \\
1 \\
- \\
43\end{array}$ & $\begin{array}{l}60 \\
- \\
- \\
8\end{array}$ & $\begin{array}{c}25 \\
- \\
- \\
5\end{array}$ & $\begin{array}{l}92,3 \\
91,3 \\
87,5 \\
91,8\end{array}$ \\
\hline SASM (140) & $\begin{array}{l}\text { CIP } \\
\text { GAT } \\
\text { MOX }\end{array}$ & $\begin{array}{c}2 \\
1 \\
14\end{array}$ & $\begin{array}{c}2 \\
10 \\
62\end{array}$ & $\begin{array}{l}12 \\
82 \\
39\end{array}$ & $\begin{array}{l}48 \\
23 \\
15\end{array}$ & $\begin{array}{c}59 \\
23 \\
9\end{array}$ & $\begin{array}{c}15 \\
- \\
-\end{array}$ & $\begin{array}{l}1 \\
\overline{1} \\
1\end{array}$ & $\begin{array}{l}\overline{1} \\
-\end{array}$ & $\begin{array}{l}- \\
- \\
-\end{array}$ & $\begin{array}{l}- \\
- \\
-\end{array}$ & $\begin{array}{l}- \\
- \\
-\end{array}$ & $\begin{array}{l}1 \\
- \\
-\end{array}$ & $\begin{array}{l}0,7 \\
0,7 \\
0,7\end{array}$ \\
\hline SCoN (176) & $\begin{array}{l}\text { CIP } \\
\text { GAT } \\
\text { MOX }\end{array}$ & $\begin{array}{l}5 \\
- \\
3\end{array}$ & $\begin{array}{c}6 \\
10 \\
55\end{array}$ & $\begin{array}{l}26 \\
68 \\
43\end{array}$ & $\begin{array}{c}56 \\
21 \\
7\end{array}$ & $\begin{array}{l}22 \\
23 \\
16\end{array}$ & $\begin{array}{l}5 \\
1 \\
7\end{array}$ & $\begin{array}{c}- \\
\underline{24} \\
\underline{33}\end{array}$ & $\begin{array}{c}\underline{6} \\
24 \\
9\end{array}$ & $\begin{array}{c}12 \\
5 \\
3\end{array}$ & $\begin{array}{c}10 \\
- \\
-\end{array}$ & $\begin{array}{c}10 \\
- \\
-\end{array}$ & $\begin{array}{l}18 \\
- \\
-\end{array}$ & $\begin{array}{l}31,8 \\
30,1 \\
25,6\end{array}$ \\
\hline SERM (90) & $\begin{array}{l}\text { CIP } \\
\text { GAT } \\
\text { MOX }\end{array}$ & $\begin{array}{l}3 \\
- \\
1\end{array}$ & $\begin{array}{c}2 \\
9 \\
33\end{array}$ & $\begin{array}{l}13 \\
30 \\
14\end{array}$ & $\begin{array}{c}23 \\
6 \\
2\end{array}$ & $\begin{array}{l}7 \\
8 \\
4\end{array}$ & $\begin{array}{l}3 \\
1 \\
5\end{array}$ & $\frac{-}{\underline{25}}$ & $\begin{array}{c}\frac{4}{16} \\
6\end{array}$ & $\begin{array}{l}7 \\
1 \\
-\end{array}$ & $\begin{array}{l}7 \\
- \\
-\end{array}$ & $\begin{array}{l}9 \\
- \\
-\end{array}$ & $\begin{array}{c}12 \\
- \\
-\end{array}$ & $\begin{array}{r}43,3 \\
40 \\
34,4\end{array}$ \\
\hline SESM (31) & $\begin{array}{l}\text { CIP } \\
\text { GAT } \\
\text { MOX }\end{array}$ & $\begin{array}{l}1 \\
- \\
1\end{array}$ & $\begin{array}{c}2 \\
- \\
11\end{array}$ & $\begin{array}{l}3 \\
21 \\
11\end{array}$ & $\begin{array}{l}13 \\
5 \\
3\end{array}$ & $\begin{array}{l}9 \\
3 \\
3\end{array}$ & $\begin{array}{l}1 \\
- \\
1\end{array}$ & $\begin{array}{l}- \\
2 \\
=\end{array}$ & $\begin{array}{l}z \\
\overline{1}\end{array}$ & $\begin{array}{l}1 \\
- \\
-\end{array}$ & $\begin{array}{l}- \\
- \\
-\end{array}$ & $\begin{array}{l}- \\
- \\
-\end{array}$ & $\begin{array}{l}1 \\
- \\
-\end{array}$ & $\begin{array}{l}6,4 \\
6,4 \\
3,2\end{array}$ \\
\hline
\end{tabular}

Subrayado: Puntos de corte $(\mathrm{mg} / \mathrm{ml})$, Negrilla: $\mathrm{CIM}_{90}$

SSARM: S. aureus resistente a meticilina; SASM: S. aureus susceptible a meticilina; SCoN: Staphylococcus coagulasa negativa; SERM: $S$. epidermidis resistente a meticilina; SESM: $S$. epidermidis susceptible a meticilina.

*CIP: ciprofloxacino; GAT: gatifloxacino; MOX: moxifloxacino; LVX: levofloxacino 
Cuadro 3. Distribución de concentraciones inhibitorias mínimas para fluoroquinolonas en enterococos.

\begin{tabular}{|c|c|c|c|c|c|c|c|c|c|c|c|c|c|c|}
\hline \multirow[t]{2}{*}{$\begin{array}{l}\text { Microorganismos } \\
\text { (n) }\end{array}$} & \multicolumn{3}{|c|}{ Antibiótico* } & \multicolumn{11}{|c|}{ Número de aislamientos con su respectiva CIM $(\mu \mathrm{g} / \mathrm{ml})$} \\
\hline & & $\leq 0,03$ & 0,06 & 0,12 & 0,25 & 0,5 & 1 & 2 & 4 & 8 & 16 & 32 & $\geq 64$ & $\% \mathbf{R}$ \\
\hline Enterococos (123) & $\begin{array}{l}\text { CIP } \\
\text { GAT } \\
\text { MOX }\end{array}$ & $\begin{array}{l}- \\
-\end{array}$ & $\begin{array}{l}- \\
- \\
2\end{array}$ & $\begin{array}{l}- \\
- \\
8\end{array}$ & $\begin{array}{r}2 \\
9 \\
44\end{array}$ & $\begin{array}{l}17 \\
55 \\
23\end{array}$ & $\begin{array}{r}37 \\
17 \\
6\end{array}$ & $\begin{array}{r}29 \\
3 \\
4\end{array}$ & $\begin{array}{l}\frac{3}{4} \\
3\end{array}$ & $\begin{array}{l}- \\
\underline{4} \\
\underline{8}\end{array}$ & $\begin{array}{r}5 \\
18 \\
22\end{array}$ & $\begin{array}{r}12 \\
12 \\
3\end{array}$ & $\begin{array}{c}18 \\
1 \\
-\end{array}$ & $\begin{array}{l}30,9 \\
28,4 \\
26,8\end{array}$ \\
\hline E. faecalis (101) & $\begin{array}{l}\text { CIP } \\
\text { GAT } \\
\text { MOX }\end{array}$ & $\begin{array}{l}- \\
- \\
-\end{array}$ & $\begin{array}{l}- \\
- \\
1\end{array}$ & $\begin{array}{l}- \\
- \\
7\end{array}$ & $\begin{array}{r}- \\
6 \\
39\end{array}$ & $\begin{array}{l}14 \\
50 \\
23\end{array}$ & $\begin{array}{r}36 \\
16 \\
4\end{array}$ & $\begin{array}{r}25 \\
3 \\
2\end{array}$ & $\begin{array}{l}\frac{1}{1} \\
2\end{array}$ & $\begin{array}{l}- \\
\underline{3} \\
\underline{5}\end{array}$ & $\begin{array}{r}3 \\
15 \\
17\end{array}$ & $\begin{array}{r}11 \\
6 \\
1\end{array}$ & $\begin{array}{c}11 \\
1 \\
-\end{array}$ & $\begin{array}{l}25,7 \\
24,7 \\
22,7\end{array}$ \\
\hline E. faecium (17) & $\begin{array}{l}\text { CIP } \\
\text { GAT } \\
\text { MOX }\end{array}$ & $\begin{array}{l}- \\
- \\
-\end{array}$ & $\begin{array}{l}- \\
- \\
1\end{array}$ & $\begin{array}{l}- \\
- \\
-\end{array}$ & $\begin{array}{l}1 \\
2 \\
2\end{array}$ & $\begin{array}{l}1 \\
2 \\
-\end{array}$ & $\begin{array}{l}1 \\
1 \\
2\end{array}$ & $\begin{array}{l}3 \\
- \\
2\end{array}$ & $\begin{array}{l}\frac{2}{3} \\
1\end{array}$ & $\begin{array}{l}- \\
\frac{1}{2}\end{array}$ & $\begin{array}{l}1 \\
2 \\
5\end{array}$ & $\begin{array}{l}1 \\
6 \\
2\end{array}$ & $\begin{array}{l}7 \\
- \\
-\end{array}$ & $\begin{array}{l}64,7 \\
52,9 \\
52,9\end{array}$ \\
\hline $\begin{array}{l}\text { Enterococos resistentes } \\
\text { a vancomicina (ERV) (12) }\end{array}$ & $\begin{array}{l}\text { CP } \\
\text { GAT } \\
\text { MOX }\end{array}$ & $\begin{array}{l}- \\
- \\
-\end{array}$ & $\begin{array}{l}- \\
- \\
-\end{array}$ & $\begin{array}{l}- \\
-\end{array}$ & - & - & $\begin{array}{l}- \\
- \\
-\end{array}$ & $\begin{array}{l}- \\
- \\
-\end{array}$ & $\begin{array}{l}= \\
- \\
-\end{array}$ & $\begin{array}{l}- \\
\overline{1} \\
1\end{array}$ & $\begin{array}{r}- \\
6 \\
10\end{array}$ & $\begin{array}{l}6 \\
6 \\
1\end{array}$ & $\begin{array}{l}6 \\
- \\
-\end{array}$ & $\begin{array}{l}100 \\
100 \\
100\end{array}$ \\
\hline E. faecalis (5) & $\begin{array}{l}\text { CIP } \\
\text { GAT } \\
\text { MOX }\end{array}$ & $\begin{array}{l}- \\
- \\
-\end{array}$ & $\begin{array}{l}- \\
- \\
-\end{array}$ & $\begin{array}{l}- \\
- \\
-\end{array}$ & $\begin{array}{l}- \\
-\end{array}$ & $\begin{array}{l}- \\
-\end{array}$ & $\begin{array}{l}- \\
- \\
-\end{array}$ & $\begin{array}{l}- \\
- \\
-\end{array}$ & $\begin{array}{l}- \\
- \\
-\end{array}$ & $\begin{array}{l}- \\
= \\
=\end{array}$ & $\begin{array}{l}- \\
4 \\
5\end{array}$ & $\begin{array}{l}5 \\
1 \\
-\end{array}$ & $\begin{array}{l}- \\
-\end{array}$ & $\begin{array}{l}100 \\
100 \\
100\end{array}$ \\
\hline E. faecium (7) & $\begin{array}{l}\text { CIP } \\
\text { GAT } \\
\text { MOX }\end{array}$ & $\begin{array}{l}- \\
-\end{array}$ & $\begin{array}{l}- \\
- \\
-\end{array}$ & $\begin{array}{l}- \\
- \\
-\end{array}$ & $\begin{array}{l}- \\
- \\
-\end{array}$ & $\begin{array}{l}- \\
- \\
-\end{array}$ & $\begin{array}{l}- \\
- \\
-\end{array}$ & $\begin{array}{l}- \\
- \\
-\end{array}$ & $\begin{array}{l}\overline{-} \\
-\end{array}$ & $\begin{array}{l}- \\
\overline{1}\end{array}$ & $\begin{array}{l}- \\
2 \\
5\end{array}$ & $\begin{array}{l}1 \\
5 \\
1\end{array}$ & $\begin{array}{l}6 \\
- \\
-\end{array}$ & $\begin{array}{l}100 \\
100 \\
100\end{array}$ \\
\hline
\end{tabular}

Subrayado: puntos de corte $(\mathrm{mg} / \mathrm{ml})$; negrilla: $\mathrm{CIM}_{90}$ ${ }^{*}$ CIP: ciprofloxacino; GAT: gatifloxacino; MOX: moxifloxacino

moxifloxacino, respectivamente (cuadro 3) (los datos de las otras especies de enterococo no se presentan). Todos los aislamientos de enterococo resistentes a vancomicina fueron resistentes a las tres fluoroquinolonas evaluadas en este estudio.

\section{Discusión}

Este trabajo es el primero en evaluar sistemáticamente la resistencia a las quinolonas de última generación en cocos Gram positivos con relevancia clínica en el ambiente hospitalario en Colombia. El neumococo es frecuentemente blanco de las quinolonas en las infecciones del sistema respiratorio $(3,6)$. De esta forma, el surgimiento de cepas de neumococo resistentes a las fluoroquinolonas es una situación preocupante, especialmente cuando se requieren alternativas orales para los aislamientos con resistencia a los macrólidos (27). El fenómeno de resistencia a las fluoroquinolonas en neumococo durante el curso de la terapia antibiótica es bien conocido y ha llevado a falla terapéutica con desenlaces fatales en pacientes hospitalizados $(28,29)$.
La prevalencia mundial de resistencia en $S$. pneumoniae a las fluoroquinolonas es inferior al $2 \%$ (3,5,6,30-34). Sin embargo, algunos estudios reportan mayores prevalencias, como el realizado en Canadá que informó un incremento en la resistencia a ciprofloxacino de más del $1 \%$ en 1997 a 4,2\% en el 2005 y a levofloxacina de 0,2 a $1,1 \%$ en el mismo periodo (23). En Asia, las tasas de resistencia a las fluoroquinolonas en el neumococo son aún mayores (24). En Hong Kong, se reportó que el $10,5 \%$ de los aislamientos recolectados entre el año 2000 y 2005 mostraban una CIM frente a ciprofloxacino mayor o igual a 4 $\mu \mathrm{g} / \mathrm{ml}(25)$ y una tasa de resistencia a levofloxacina de $13,3 \%$, que se incrementó a $27,3 \%$ en aislamientos resistentes a la penicilina (35). España, en el 2001, informó una tasa de resistencia del $7 \%$ de aislamientos con una $\mathrm{CIM} \geq 4 \mu \mathrm{g} / \mathrm{ml}$ para ciprofloxacino (26). Por el contrario, en Estados Unidos la prevalencia de resistencia a las fluoroquinolonas es inferior al $2 \%(36,37)$.

De acuerdo con los datos obtenidos del sistema de vigilancia PROTEKT, de 3.362 aislamientos 
de S. pneumoniae recolectados durante 1999-2000 (38) de diferentes partes del mundo, se evidenció que los valores de $\mathrm{CIM}_{50}$ y $\mathrm{CIM}_{90}$ para la levofloxacina fueron de $1 \mu \mathrm{g} / \mathrm{ml}$ para todos los países participantes en el estudio, con la notable excepción de Hong Kong, donde la $\mathrm{CIM}_{90}$ fue de $16 \mu \mathrm{g} / \mathrm{ml}$ (38). Estos datos son consistentes con nuestros resultados en donde se encontró que sólo un aislamiento de neumococo presentó una CIM incrementada para las fluoroquinolonas de última generación (moxifloxacino y gatifloxacino). El hallazgo de altos niveles de resistencia en este aislamiento sugiere la presencia de varias mutaciones en los genes gyrA y parC.

No obstante, es importante resaltar que la $\mathrm{CIM}_{90}$ para el ciprofloxacino fue relativamente alta $(\geq 4 \mu \mathrm{g} /$ $\mathrm{ml}$ ) en los aislamientos colombianos de neumococo (esta característica fenotípica no tuvo una relación importante con la resistencia a penicilina o ceftriaxona). Este hallazgo de elevada CIM frente al ciprofloxacino, podría sugerir que las "mutaciones de primer paso", que no afectan los compuestos de última generación, podrían estar presentes en estos aislamientos. La presencia de estas mutaciones puede eventualmente predisponer al desarrollo de resistencia a fluoroquinolonas de última generación si estos antibióticos son usados indiscriminadamente.

Nuestros datos confirman que la resistencia a las fluoroquinolonas en el neumococo continúa siendo baja en Colombia y permite concluir que las fluoroquinolonas de última generación continúan siendo una alternativa importante para el tratamiento de infecciones respiratorias por neumococo.

Es importante anotar que nuestro grupo ha demostrado que la resistencia a los macrólidos en los mismos aislamientos es también baja $(3,3 \%)$, aunque se ha incrementado ligeramente en los últimos años (18). Estos datos nos permiten hacer la recomendación de que las fluoroquinolonas deben reservarse para el manejo de la neumonía en casos en los que se evidencian altos niveles de resistencia a $\beta$-lactámicos y macrólidos o en caso de alergia a estos compuestos.

Por el contrario, con el neumococo, las tasas de resistencia a las fluoroquinolonas reportadas en todo el mundo en Staphylococcus spp. y Entero- coccus spp. son elevadas. En relación con $S$. aureus, desde la introducción del ciprofloxacino a la práctica clínica, rápidamente emergieron cepas resistentes, y se han reportado prevalencias del $15 \%$ al $50 \%$ en países europeos y Egipto (39-41) y aumentando dramáticamente en cepas de $S$. aureus resistentes a meticilina, en las que llegan a ser superiores al $80 \%$ (41-45). En estafilococos coagulasa negativa se reporta una prevalencia de resistencia a fluoroquinolonas entre $30 \%$ y $60 \%$ $(39,41,45)$ y para el enterococo la resistencia es superior al $30 \%$ en Latinoamérica (46). Se ha observado, además, que los aislamientos de $E$. faecium presentan prevalencias de resistencia a las fluoroquinolonas hasta del $89 \%$ (37), las cuales incrementan al $100 \%$ en los aislamientos de enterococo resistentes a los glicopéptidos (47).

Nuestros resultados indican que en nuestro país está ocurriendo un incremento en la resistencia a las fluoroquinolonas en $S$. aureus resistentes a la meticilina en los últimos años. En un programa de vigilancia de $S$. aureus resistentes a la meticilina en hospitales de Bogotá realizado entre 1996 y 1998, se demostró que la resistencia a ciprofloxacino era del $58 \%$; este valor se incrementó al $98,5 \%$ en el periodo comprendido entre el 2001 y el $2003(10,19)$.

La explicación más probable para este notorio incremento en tan corto tiempo es el cambio de clones que ha ocurrido en la genética de la población de $S$. aureus resistentes a la meticilina (19). El clon predominante circulante en Colombia entre 1996 y 1998 ha sido reemplazado por un nuevo clon (designado clon chileno) (48). Este grupo de clones es el que circula actualmente en hospitales colombianos y se caracteriza por altos niveles de resistencia a macrólidos y fluoroquinolonas (19).

Una posible explicación para este fenómeno es que el uso indiscriminado de fluoroquinolonas en nuestro país, particularmente aquéllas con reducida actividad contra bacterias Gram positivas, como el ciprofloxacino, seleccione cepas resistentes que se establecen fuertemente en el medio. Los estudios recientes han establecido una asociación entre el uso masivo de fluoroquinolonas y la diseminación de $S$. aureus resistentes a la meticilina y cepas virulentas de 
Clostridium difficile en los hospitales canadienses $(49,50)$ y un fenómeno similar podría estar ocurriendo en Colombia.

El hallazgo de altos niveles de resistencia a las quinolonas de última generación -que poseen una actividad incrementada contra Gram positivosen $S$. aureus resistentes a la meticilina, como el gatifloxacino y el moxifloxacino, en un país donde su uso no es común por el alto costo, sustenta la hipótesis de que la presión selectiva ha sido ejercida por compuestos con menor actividad frente a $S$. aureus resistentes a la meticilina, como el ciprofloxacino, presentándose de esta forma resistencia cruzada como se ha descrito previamente (51). Estos datos también nos permiten afirmar que es evidente que las fluoroquinolonas no son una alternativa útil en el tratamiento de las infecciones por $S$. aureus resistentes a la meticilina en Colombia.

En estafilococos coagulasa negativa, la resistencia a las quinolonas osciló entre $25 \%$ y $32 \%$ para los diferentes compuestos (cuadro 2), con un incremento notable en los aislamientos de $S$. epidermidis resistentes a la meticilina -con una tasa de resistencia a ciprofloxacino de $43,3 \%-$ en comparación con los $S$. epidermidis susceptibles a la meticilina $(6,4 \%)$. Estos datos concuerdan con lo reportado previamente en la literatura $(39,41,45)$.

Como se indicó anteriormente, nuestros datos sobre enterococo concuerdan con el hecho de que los todos los aislamientos resistentes a la vancomicina mostraron resistencia a todas las fluoroquinolonas, inclusive los compuestos de última generación $(41,46)$.

Recientemente, se reportó el hallazgo de resistencia a fluoroquinolonas mediada por qnren una cepa de E. faecalis (9), mecanismo que se había descrito únicamente en enterobacterias $(7,52)$. La investigación de la presencia de qnr en cepas de enterococo resistentes a las fluoroquinolonas en Colombia está siendo investigada y será el objeto de una comunicación ulterior.

En conclusión, nuestros hallazgos indican que las fluoroquinolonas de última generación conservan un buen perfil de susceptibilidad en neumococo y se presentan como alternativas útiles en el tratamiento de infecciones respiratorias causadas por este microorganismo. No obstante, debido a la rapidez en el desarrollo de la resistencia mediada por mutaciones en el blanco molecular de la quinolonas, su uso debe ser restringido a aquellas situaciones en donde otros antibióticos ( $\beta$-lactámicos y macrólidos) no puedan ser utilizados. Por el contrario, la actividad de las fluoroquinolonas es muy pobre contra las cepas de enterococo y estafilococo, particularmente $S$. aureus resistentes a la meticilina hospitalarios. El valor de estos compuestos para aislamientos asociados a la comunidad ( $S$. aureus resistentes a meticilina-AC), los cuales han emergido en Colombia desde el 2005 (53), está por determinarse.

\section{Conflicto de intereses}

Los autores declaramos que durante la realización del presente trabajo no existió conflicto de interés financiero, político, académico, personal, ni de ningún tipo que pudiera haber afectado los resultados del mismo.

\section{Financiación}

Este trabajo fue financiado por el Instituto Colombiano para el desarrollo de la Ciencia y Tecnología "Francisco José de Caldas", Colciencias, código № 2104-04-12685, por el Grupo de Microbiología del Instituto Nacional de Salud y el Instituto de Genética Molecular Bacteriana, Universidad El Bosque.

\section{Agradecimientos}

A Bayer Corporation por la donación del antibiótico moxifloxacina. A Germán Contreras, Leonardo Cortés, Tatiana Alba, Camilo Ardila y Gustavo Huertas por su asistencia técnica.

\section{Referencias}

1. Lesher GY, Froelich EJ, Gruett MD, Bailey JH, Brundage RP. 1,8-Naphthyridine derivates: a new class of chemotherapeutic agents. J Med Pharm Chem. 1962;5:1063-5.

2. Hopper DC. New uses for new and old quinolones and challenge of resistance. Clin Infect Dis. 2000;30:243-54.

3. Hooper DC. Fluoroquinolone resistance among Grampositive cocci. Lancet Infect Dis. 2002;2:530-8. 
4. Saravolatz LD, Leggett J. Gatifloxacin, gemifloxacin, and moxifloxacin: the role of 3 newer fluoroquinolones. Clin Infect Dis. 2003;37:1210-5.

5. Jacoby GA. Mechanisms of resistance to quinolones. Clin Infect Dis. 2005;41:S120-6.

6. Schmitz FJ, Higgins PG, Mayer S, Fluit AC, Dalhof A. Activity of quinolones against Gram positive cocci: Mechanisms of drug action and bacterial resistance. Eur J Clin Microbiol Infect Dis. 2002;21:647-59.

7. Tran JH, Jacoby GA. Mechanism of plasmid-mediated quinolone resistance. Proc Natl Acad Sci USA. 2002;99: 5638-42.

8. Ruiz J. Mechanisms of resistance to quinolones: target alterations, decreased accumulation and DNA gyrase protection. J Antimicrob Chemother. 2003;51:1109-17.

9. Arsene S, Leclercq R. Role of a qnr-like gene in the intrinsic resistance of Enterococcus faecalis to fluoroquinolones. Antimicrob Agents Chemother. 2007;51:3254-8.

10. Arias CA, Reyes J, Zuñiga M, Cortés L, Cruz C, Rico CL, et al. Multicentre surveillance of antimicrobial resistance in enterococci and staphylococci from Colombian hospitals, 2001-2002. J Antimicrob Chemother. 2003;51:59-68.

11. Álvarez C, Cortés J, Arango A, Correa C, Leal A, Grupo para el Control de la Resistencia Bacteriana en Bogotá. Anti-microbial resistance in intensive care units in Bogotá, Colombia, 2001-2003. Rev Salud Pública (Bogotá). 2006;8(Suppl.1):86-101 .

12. Grupo de Microbiología. Streptococcus pneumoniae aislamientos invasores. [Consultado: 19 de septiembre 2006].Disponible en: http://www.ins.gov.co/pdf_investiga/ Microbiologia_spn_005.pdf.

13. Organización Panamericana de la Salud. Programa de Vigilancia de los serotipos y resistencia antimicrobiana de Streptococcus pneumonaie y Haemophilus influenzae. Manual de Procedimientos. [Consultado: 3 de marzo de 2006]. Disponible en:http:/ /www.paho.org/Spanish/AD/THS/EV/LABS-manualvigilancia-serotipos.pdf.

14. Agudelo $\mathrm{Cl}$, Moreno J, Sanabria OM, Ovalle MV, Di Fabio JL, Castañeda E, et al. Streptococcus pneumoniae: evolución de los serotipos y los patrones de susceptibilidad antimicrobiana en aislamientos invasores en 11 años de vigilancia en Colombia (19942004). Biomédica. 2006;26:234-49.

15. Martineau F, Picard FJ, Lansac N, Ménard C, Roy $\mathrm{PH}$, Ouellette $\mathbf{M}$, et al. Correlation between the resistance genotype determined by multiplex PCR assays and the antibiotic susceptibility patterns of Staphylococcus aureus and Staphylococcus epidermidis. Antimicrob Agents Chemother. 2000;44: 231-8.

16. Dutka-Malen S, Evers S, Courvalin P. Detection of glycopeptide resistance genotypes and identification to the species level of clinically relevant enterococci by PCR. J Clin Microbiol. 1995;33:24-7.

17. Woodford N, Egelton CM, Morrison D. Comparison of PCR with phenotypic methods for the speciation of enterococci. Adv Exp Med Biol. 1997;418:405-8.

18. Reyes J, Hidalgo M, Díaz L, Rincón S, Moreno J, Vanegas $\mathbf{N}$, et al. Characterization of macrolide resistance in Gram-positive cocci from Colombian hospitals: a countrywide surveillance. Int $\mathrm{J}$ Infect Dis. 2007;11:329-36.

19. Cruz C, Moreno J, Renzoni A, Hidalgo M, Reyes J, Schrenzel J, et al. Tracking methicillin-resistant Staphylococcus aureus clones in Colombian hospitals over 7 years (1996-2003): emergence of a new dominant clone. Int J Antimicrob Agents. 2005;26:457-62.

20. Clinical and Laboratory Standards Institute. Performance Standards for Antimicrobial Susceptibility Testing: Fifteenth Informational Supplement. M7-A6 Methods for dilution antimicrobial susceptibility test for bacteria that grow aerobically: Approved Standard. Sixth Edition. Wayne, Pennsylvania: CLSI; 2005.

21. Clinical and Laboratory Standards Institute. Performance Standards for Antimicrobial Susceptibility Testing: Fifteenth Informational Supplement. M2-A8 Performance standards for antimicrobial disk susceptibility test; Approved standard. Eighth edition. Wayne, Pennsylvania: CLSI; 2005.

22. López H, Sader H, Amábile C, Pedreira W, MuñozBellido JL, García-Rodríguez JA, et al. In vitro activity of moxifloxacin against respiratory pathogens in Latin America. Rev Esp Quimioter. 2002;15:325-34

23. Adam HJ, Schurek KN, Nichol KA, Hoban CJ, Baudry TJ, Laing DJ, et al. Molecular characterization of increasing fluoroquinolone resistance in Streptococcus pneumoniae in Canada 1997 to 2005. Antimicrob Agents Chemother. 2007;51:198-207.

24. Song JH, Jung SI, Ko KS, Kim NY, Son JS, Chang $\mathbf{H H}$, et al. High prevalence of antimicrobial resistance among clinical Streptococcus pneumoniae isolates in Asia (an ANSORP study). Antimicrob Agents Chemother. 2004;48:2101-7.

25. Ip M, Chau SSL, Chi F, Cheuk ES, Ma H, Lai RW, et al. Longitudinally tracking fluoroquinolone resistance and its determinants in penicillin-susceptible and nonsusceptible Streptococcus pneumoniae isolates in Hong Kong, 2000-2005. Antimicrob Agents Chemother. 2007; 51:2192-4.

26. Pérez-Trallero E, Fernández-Mazarrasa C, GarcíaRey C, Bouza E, Aguilar L, García-de-Lomas J, et al. Spanish Surveillance Group for Respiratory Pathogens. Antimicrobial susceptibilities of 1,684 Streptococcus pneumoniae and 2,039 Streptococcus pyogenes isolates and their ecological relationships: results of a 1-year (1998-1999) multicenter surveillance 
study in Spain. Antimicrob Agents Chemother. 2001;45: 3334-40.

27. Mandell LA, Bartlett JG, Dowell SF, File TM, Musher DM, Whitney C, et al. Update of practice guidelines for the management of community-acquired pneumonia in immunocompetent adults. Clin Infect Dis. 2003;37: 1405-33.

28. Ambrose PG, Bast D, Doern GV, lannini PB, Jones $\mathbf{R N}$, Klugman KP, et al. Fluoroquinolone-resistant Streptococcus pneumoniae, an emerging but unrecognized public health concern: is it time to resight the goalposts? Clin Infect Dis. 2004;39:1554-6.

29. Fuller JD, Low D. A review of Streptococcus pneumoniae infection treatment failures associated with fluoroquinolone resistance. Clin Infect Dis. 2005;41: 118-21.

30. Legg JM, Bint AJ. Will pneumococci put quinolones in their place? J Antimicrob Chemother. 1999;44:425-7.

31. Niederman MS. Challenges in the management of community-acquired pneumonia: the role of quinolones and moxifloxacin. Clin Infect Dis. 2005;41:S158-66.

32. Dobay O, Rozgonyi F, Ghidán A, Matuz M, Nagy K, Amyes SG. The first steps towards fluoroquinolone resistance in Hungarian pneumococci. J Chemother. 2006;18:624-7.

33. Dias R, Louro D, The Antimicrobial Resistance Surveillance Program in Portugal, Caniça M. Antimicrobial susceptibility of invasive Streptococcus pneumoniae isolates in Portugal over an 11-Year Period. Antimicrob Agents Chemother. 2006;50:2098-105.

34. Jones ME, Blosser-Middleton RS, Thornsberry C, Karlowsky JA, Sahm DF. The activity of levofloxacin and other antimicrobials against clinical isolates of Streptococcus pneumon iae collected worldwide during 1999-2002. Diagn Microbiol Infect Dis. 2003;47:57986.

35. Ho PL, Yung RW, Tsang DN, Que TL, Ho M, Seto WH. Increasing resistance of Streptococcus pneumoniae to fluoroquinolones: results of a Hong Kong multicentre study in 2000. J Antimicrob Chemother. 2001; 48:659-65.

36. Richter SS, Heilmann KP, Beekmann SE, Miller NJ, Rice CL, Doern GV. The molecular epidemiology of Streptococcus pneumoniae with quinolone resistant mutations. Clin Infect Dis. 2005;40:225-35.

37. Karlowsky JA, Jones ME, Draghi DC, Thornsberry C, Sahm DF, Volturo GA. Prevalence and antimicrobial susceptibilities of bacteria isolated from blood cultures of hospitalized patients in the United States in 2002. Ann Clin Microbiol Antimicrob. 2004;10:3-7.

38. Canton R, Morosini M, Enright MC, Morrissey I. Worldwide incidence, molecular epidemiology and mutations implicated in fluoroquinolone-resistant
Streptococcus pneumoniae: data from the global PROTEKT surveillance programme. J Antimicrob Chemother. 2003;52:944-52.

39. Andrews J, Ashby J, Jevons G, Marshall T, Lines $\mathbf{N}$, Wise R. A comparison of antimicrobial resistance rates in Gram-positive pathogens isolated in the UK from October 1996 to January 1997 and October 1997 to January 1998. J Antimicrob Chemother. 2000;45: 285-93.

40. EI Kholy A, Baseem H, Hall GS, Procop GW, Longworth DL. Antimicrobial resistance in Cairo, Egypt 1999-2000: a survey of five hospitals. J Antimicrob Chemother. 2003;51:625-30.

41. Decousser JW, Pina P, Picot F, Delalande C, Pangon $\mathbf{B}$, Courvalin $\mathbf{P}$, et al. Frequency of isolation and antimicrobial susceptibility of bacterial pathogens isolated from patients with bloodstream infections: a French prospective national survey. J Antimicrob Chemother. 2003;51:1213-22.

42. Blumberg HM, Rimland D, Carroll DJ, Terry $\mathbf{P}$, Wachsmuth IK. Rapid development of ciprofloxacin resistance in methicillin-susceptible and methicillinresistant Staphylococcus aureus. J Infect Dis. 1991; 163:1279-85.

43. Zangrillo A, Landoni G, Fumagalli L, Bove T, Bellotti F, Sottocorna O, et al. Methicillin-resistant Staphylococcus species in a cardiac surgical intensive care unit: a 5-year experience. J Cardiothorac Vasc Anesth. 2006;20:31-7.

44. Klevens M. Changes in the epidemiology of methicillinresistant Staphylococcus aureus in intensive care units in US hospitals, 1992-2003. Clin Infect Dis. 2006;42: 389-91.

45. Reynolds R, Potz N, Colman M, Williams A, Livermore D, MacGowan A, et al. Antimicrobial susceptibility of the pathogens of bacteraemia in the UK and Ireland 2001-2002: the BSAC Bacteraemia Resistance Surveillance Programme. J Antimicrob Chemother. 2004:53:1018-32.

46. Andrade SS, Sader HS, Jones RN, Pereira AS, Pignatari AC, Gales AC. Increased resistance to firstline agents among bacterial pathogens isolated from urinary tract infections in Latin America: time for local guidelines? Mem Inst Oswaldo Cruz. 2006;101:741-8.

47. Deshpande LM, Fritsche TR, Moet GJ, Biedenbach DJ, Jones RN. Antimicrobial resistance and molecular epidemiology of vancomycin-resistant enterococci from North America and Europe: a report from the SENTRY antimicrobial surveillance program. Diagn Microbiol Infect Dis. 2007;58:163-70.

48. Aires De Sousa M, Miragaia M, Sanches IS, Avila S, Adamson I, Casagrande ST, et al. Three-year assessment of methicillin-resistant Staphylococcus aureus clones in Latin America from 1996 to 1998. J Clin Microbiol. 2001;39:2197-205. 
49. Weber SG, Gold HS, Hooper DC, Karchmer AW, Carmeli Y. Fluoroquinolones and the risk for methicillinresistant Staphylococcus aureus in hospitalized patients. Emerg Infect Dis. 2003;9:1415-22.

50. Loo VG, Poirier L, Miller MA, Oughton M, Libman MD, Michaud S, et al. A predominantly clonal multiinstitutional outbreak of Clostridium difficile-associated diarrhea with high morbidity and mortality. $\mathrm{N}$ Engl J Med. 2005;8;353:2442-9.
51. Sanders CC. Mechanisms responsible for crossresistance and dichotomous resistance among the quinolones. Clin Infect Dis. 2001;32(Suppl.1):S1-8.

52. Rodríguez-Martínez JM. Mechanisms of plasmidmediated resistance to quinolones. Enferm Infecc Microbiol Clin. 2005;23:25-31.

53. Álvarez CA, Barrientes OJ, Leal AL, Contreras GA, Barrero L, Rincón S, et al. Community-associated methicillin-resistant Staphylococcus aureus, Colombia. Emerg Infect Dis. 2006;12:2000-1. 